PROCEEDINGS OF THE

AMERICAN MATHEMATICAL SOCIETY

Volume 127, Number 10, Pages 2841-2850

S 0002-9939(99)05097-2

Article electronically published on June 17, 1999

\title{
RESIDUALLY FINITE, CONGRUENCE MEET-SEMIDISTRIBUTIVE VARIETIES OF FINITE TYPE HAVE A FINITE RESIDUAL BOUND
}

\author{
KEITH A. KEARNES AND ROSS WILLARD
}

(Communicated by Lance W. Small)

\begin{abstract}
We show that a residually finite, congruence meet-semidistributive variety of finite type is residually $<N$ for some finite $N$. This solves Pixley's problem and a special case of the restricted Quackenbush problem.
\end{abstract}

\section{INTRODUCTION}

This paper concerns varieties of general algebras and their residual character. By an algebra $\mathbf{A}$ we mean any nonempty set $A$ together with a family of operations $f_{i}$ : $A^{n_{i}} \rightarrow A(i \in I)$ of various arities $n_{i}<\omega$. A variety is any class of algebras definable by a set of equational laws in a specified family of operation symbols. Examples are the variety of commutative semigroups (one binary operation, commutative and associative laws) and the variety of Boolean algebras (two binary operations, one unary operation, two nullary operations, a well-known list of laws).

Of central importance when studying a variety are the subdirectly irreducible members (the SI's). These are the algebras $\mathbf{A}$ in the variety which are residually irreducible in the following sense: there exist $a, b \in A$ with $a \neq b$ such that any homomorphism $h$ with domain $\mathbf{A}$ and satisfying $h(a) \neq h(b)$ must be an embedding. For example, the 2-element Boolean algebra is the unique subdirectly irreducible Boolean algebra. By contrast, in the variety of commutative semigroups there are $2^{\kappa}$ SI's of every infinite cardinality $\kappa$, and they are unclassifiable in the sense that they interpret all posets (and hence all first-order structures in any finite language - see [5, Theorem 5.5.2]).

A variety is:

1. residually small if there is a cardinal greater than the cardinality of every SI in the variety;

2. residually finite if every SI in the variety is finite;

3. residually $<N$ if every $\mathrm{SI}$ in the variety has cardinality less than $N$.

V(A) denotes the smallest variety containing the algebra A. In 1971, R. Quackenbush posed the following problem [10].

Received by the editors January 6, 1998.

1991 Mathematics Subject Classification. Primary 08B26, 08B10.

Key words and phrases. Congruence distributive, semidistributive, residually finite, variety.

The second author gratefully acknowledges the support of the NSERC of Canada.

(C)1999 American Mathematical Society 
The Quackenbush Problem. If $\mathbf{A}$ is a finite algebra and $\mathbf{V}(\mathbf{A})$ is residually finite, must $\mathbf{V}(\mathbf{A})$ be residually $<N$ for some $N<\omega$ ?

This question was finally answered negatively in the Fall of 1993 by R. McKenzie [9] using an ingenious construction involving infinitely many operations on a 4-element set. The question remains open, and plausible, when restricted to finite algebras having only finitely many operations. We shall call this version the Restricted Quackenbush Problem.

A variety $\mathcal{V}$ is congruence distributive if, for every member $\mathbf{A} \in \mathcal{V}$, the lattice of congruence relations of $\mathbf{A}$ ( = equivalence relations on $A$ which are compatible with the operations of $\mathbf{A}$ ) satisfies the distributive law. Congruence distributive varieties are relatively well-understood; for example, the Quackenbush Problem is settled affirmatively when $\mathbf{V}(\mathbf{A})$ is congruence distributive, by an old observation of A. Foster and A. Pixley [3, Theorem 2.5]. In 1984, Pixley asked the following question (see also [6, Problem 4.4]).

Pixley's Problem. If $\mathcal{V}$ is a residually finite, congruence distributive variety of finite type (that is, having only finitely many operations), must $\mathcal{V}$ be residually $<N$ for some $N<\omega$ ?

At the time this problem was posed, it pinpointed the only gap in our knowledge about the residual character of congruence distributive varieties. In the meantime it has been solved for affine complete congruence distributive varieties (K. Kaarli and A. Pixley [6]), for varieties of lattices (R. Freese and J. B. Nation, unpublished), and for varieties of lattice-based algebras (R. McKenzie, unpublished).

Let us call a variety a Pixley variety if it has finite type and is residually finite but not residually $<N$ for any $N<\omega \cdot{ }^{1}$ In this paper we prove that there is no Pixley variety whose congruence lattices satisfy the meet-semidistributive implication

$$
x \wedge y=x \wedge z \quad \longrightarrow \quad x \wedge y=x \wedge(y \vee z) .
$$

As meet-semidistributivity follows from distributivity, this settles Pixley's Problem. It also yields a positive answer to the Restricted Quackenbush Problem whenever the variety $\mathbf{V}(\mathbf{A})$ is congruence meet-semidistributive; this is the case, e.g., whenever the operations of $\mathbf{A}$ include a semilattice operation (more generally, see [4, Theorem 9.10]). Our arguments are based on an analysis of the combinatorial properties of principal congruence generation in congruence meet-semidistributive varieties. Virtually the same combinatorial properties were discovered and used by K. Baker in [1] to prove a finite basis theorem.

\section{Arrows}

Suppose $\mathbf{A}$ is an algebra. Following [1], by a basic translation of $\mathbf{A}$ we mean any unary polynomial of the form $F\left(a_{1}, \ldots, a_{i-1}, x, a_{i+1}, \ldots, a_{n}\right)$ where $F$ is an $n$-ary fundamental operation of $\mathbf{A}, 1 \leq i \leq n$, and the $a_{j}$ 's are any elements of $A$. A $k$-translation of $\mathbf{A}$ is a unary polynomial of $\mathbf{A}$ which can be expressed as the composition of $k$ or fewer basic translations. In particular, the identity map $\operatorname{id}_{A}$ is the unique 0-translation of $\mathbf{A}$.

$A^{(2)}$ denotes the set of all 2-element subsets of $A$. If $\{a, b\},\{c, d\} \in A^{(2)}$ and $k<$ $\omega$, then we write $\{a, b\} \rightarrow_{k}\{c, d\}$ to mean that there exists a $k$-translation $f$ such

\footnotetext{
${ }^{1}$ An example of a Pixley variety is the variety with two unary operations $f, g$ defined by the laws $f g(x)=x$ and $g f(x)=x$.
} 
that $\{f(a), f(b)\}=\{c, d\}$. If $X \subseteq A^{(2)}$, then $X \rightarrow_{k}\{c, d\}$ means $\{a, b\} \rightarrow_{k}\{c, d\}$ for all $\{a, b\} \in X$.

Similarly, if $k, n<\omega$, then we define $\{a, b\} \Rightarrow_{k, n}\{c, d\}$ to mean that there exists a sequence $c=c_{0}, c_{1}, \ldots, c_{n}=d$ such that for each $i<n$, either $c_{i}=c_{i+1}$ or $\{a, b\} \rightarrow_{k}\left\{c_{i}, c_{i+1}\right\}$. The notation $\{a, b\} \Rightarrow_{k}\{c, d\}$ means $\{a, b\} \Rightarrow_{k, n}\{c, d\}$ for some $n<\omega$. If $X \subseteq A^{(2)}$, then $X \Rightarrow_{k, n}\{c, d\}$ and $X \Rightarrow_{k}\{c, d\}$ mean the obvious things, namely, that $\{a, b\} \Rightarrow_{k, n}\{c, d\}$ (respectively, $\{a, b\} \Rightarrow_{k}\{c, d\}$ ) for all $\{a, b\} \in X$. Note that by Mal'cev's description of principal congruences, if $\{a, b\},\{c, d\} \in A^{(2)}$, then $(c, d) \in \mathrm{Cg}^{\mathbf{A}}(a, b)$ if and only if $\{a, b\} \Rightarrow_{k}\{c, d\}$ for some $k<\omega$. Moreover, if the language of $\mathbf{A}$ is finite, then for all $k, n<\omega$ there is a first-order formula $\pi(x, y, z, w)$ (a principal congruence formula in the sense of [11]) which defines the relation $\{x, y\} \Rightarrow_{k, n}\{z, w\}$ in all algebras of the same type as $\mathbf{A}$.

The relations $\rightarrow_{k}$ and $\Rightarrow_{k, n}$ have the following properties.

1. $\rightarrow_{k}$ and $\Rightarrow_{k, 1}$ mean the same thing.

2. If $\{a, b\} \Rightarrow_{k, m}\{c, d\} \Rightarrow_{\ell, n}\{r, s\}$, then $\{a, b\} \Rightarrow_{k+\ell, m n}\{r, s\}$. In other words, compositions of $\Rightarrow_{x, y}$ are additive in $x$ and multiplicative in $y$.

3. If $\{a, b\} \rightarrow_{k+\ell}\{c, d\}$, then there exist $u, v$ such that $\{a, b\} \rightarrow_{k}\{u, v\} \rightarrow_{\ell}$ $\{c, d\}$.

\section{Sequence Lemmas}

Congruence meet-semidistributivity was shown to be a weak Mal'cev property by G. Czédli [2]. That is, he found an infinite sequence $\mathcal{C}_{2}, \mathcal{C}_{3}, \ldots$ of Mal'cev properties such that a variety is congruence meet-semidistributive if and only if it satisfies $\mathcal{C}_{m}$ for all $m \geq 2$. Later, it was shown by Kearnes and Á. Szendrei [7] and P. Lipparini [8] that $\mathcal{C}_{2}$ already characterizes congruence meet-semidistributivity. More recently, using the Mal'cev condition directly derivable from $\mathcal{C}_{2}$, Willard proved the following.

Theorem $3.1([12])$. For a variety $\mathcal{V}$, the following are equivalent:

(1) $\mathcal{V}$ is congruence meet-semidistributive.

(2) There exists a finite family $\left\{\left\langle s_{i}(x, y, z), t_{i}(x, y, z)\right\rangle: 0 \leq i \leq p\right\}$ of pairs of ternary terms such that

$$
\begin{aligned}
\mathcal{V} & =s_{i}(x, y, x) \approx t_{i}(x, y, x), \quad(0 \leq i \leq p), \\
\mathcal{V} & =\forall x y\left(x=y \leftrightarrow \bigwedge_{i=0}^{p}\left[s_{i}(x, x, y)=t_{i}(x, x, y) \leftrightarrow s_{i}(x, y, y)=t_{i}(x, y, y)\right]\right)
\end{aligned}
$$

For example, if $\mathcal{V}$ is a congruence distributive variety with Jónsson terms $d_{0}(x, y, z), \ldots, d_{k}(x, y, z)$, then an appropriate family of pairs is $\left\{\left\langle d_{i}(x, y, z)\right.\right.$, $\left.\left.d_{i+1}(x, y, z)\right\rangle: i<k\right\}$. If $\mathcal{V}$ has a semilattice term operation $x y$, then an appropriate family of pairs is $\{\langle x y z, x y\rangle,\langle x y z, y z\rangle\}$.

The next two lemmas are taken from [12]; their counterparts in the congruence distributive case are [1, Lemmas 5.5 and 5.3].

Lemma 3.2 (Single-sequence lemma). Suppose the variety $\mathcal{V}$ is congruence meetsemidistributive with terms $s_{i}(x, y, z), t_{i}(x, y, z)$ witnessing Theorem 3.1 (2). Suppose moreover that each $s_{i}, t_{i}$ is a fundamental operation symbol in the language of $\mathcal{V}$. Then the following is true: if $\mathbf{A} \in \mathcal{V}$ and $a=a_{0}, a_{1}, \ldots, a_{n}=b$ is $a$ sequence in $A$ with $a \neq b$, then there exist $\{c, d\} \in A^{(2)}$ and $i<n$ such that $\left\{a_{i}, a_{i+1}\right\} \Rightarrow_{1,2}\{c, d\}$ and $\{a, b\} \Rightarrow_{1,2}\{c, d\}$. 
Lemma 3.3 (Multi-sequence lemma). With the same assumptions as before, the following is true: if $\mathbf{A} \in \mathcal{V},\{a, b\} \in A^{(2)}$, and $S_{1}, \ldots, S_{N}$ are sequences from a to $b$, where $S_{i}=\left(a_{0}^{i}, a_{1}^{i}, \ldots, a_{\lambda(i)}^{i}\right)$ with $a_{0}^{i}=a$ and $a_{\lambda(i)}^{i}=b$ for each $i=1, \ldots, N$, then there exist $\{u, v\} \in A^{(2)}$ and, for each $i$, a 'key link' $\left\{a_{\sigma(i)}^{i}, a_{\sigma(i)+1}^{i}\right\}$ of distinct adjacent elements of $S_{i}$, which we shall rename $\left\{a^{i}, b^{i}\right\}$, such that $\left\{a^{i}, b^{i}\right\} \Rightarrow_{N, 2^{N}}$ $\{u, v\}$ for each $i$ and $\{a, b\} \Rightarrow_{N, 2^{N}}\{u, v\}$.

Proof of Lemma 3.2. As $a \neq b$, Theorem 3.1 gives the existence of $j \leq p$ such that $\neg\left[s_{j}(a, a, b)=t_{j}(a, a, b) \leftrightarrow s_{j}(a, b, b)=t_{j}(a, b, b)\right]$. Suppose for concreteness that $s_{j}(a, a, b)=t_{j}(a, a, b)$ while $s_{j}(a, b, b) \neq t_{j}(a, b, b)$. As $a=a_{0}$ while $b=a_{n}$, there must exist $i<n$ such that $s_{j}\left(a, a_{i}, b\right)=t_{j}\left(a, a_{i}, b\right)$ while $s_{j}\left(a, a_{i+1}, b\right) \neq$ $t_{j}\left(a, a_{i+1}, b\right)$. Let $c=s_{j}\left(a, a_{i+1}, b\right), d=t_{j}\left(a, a_{i+1}, b\right), u=s_{j}\left(a, a_{i}, b\right)$, and $v=$ $s_{j}\left(a, a_{i+1}, a\right)$. Define $f_{1}(x)=s_{j}(a, x, b), f_{2}(x)=t_{j}(a, x, b), g_{1}(x)=s_{j}\left(a, a_{i+1}, x\right)$, and $g_{2}(x)=t_{j}\left(a, a_{i+1}, x\right)$. $f_{1}, f_{2}, g_{1}, g_{2}$ are basic translations of $\mathbf{A}$ which satisfy

$$
\begin{aligned}
\left\{f_{1}\left(a_{i}\right), f_{1}\left(a_{i+1}\right)\right\} & =\{c, u\} \\
\left\{f_{2}\left(a_{i}\right), f_{2}\left(a_{i+1}\right)\right\} & =\{u, d\} \quad \text { by assumption, } \\
\left\{g_{1}(a), g_{1}(b)\right\} & =\{c, v\}, \\
\left\{g_{2}(a), g_{2}(b)\right\} & =\{v, d\} \quad \text { using the identity } s_{j}(x, y, x) \approx t_{j}(x, y, x) .
\end{aligned}
$$

Thus $\left\{a_{i}, a_{i+1}\right\} \Rightarrow_{1,2}\{c, d\}$ and $\{a, b\} \Rightarrow_{1,2}\{c, d\}$.

Proof of Lemma 3.3. The proof is virtually the same as Baker's proof of his original multisequence lemma [1, Lemma 5.3]. Argue by induction on $N$. If $N=1$, then the claim is Lemma 3.2. If $N>1$, apply the claim to the sequences $S_{1}, \ldots, S_{N-1}$ to get $\{c, d\} \in A^{(2)}$ and key links $\left\{a^{1}, b^{1}\right\}, \ldots,\left\{a^{N-1}, b^{N-1}\right\}$ such that

$$
\begin{array}{rll}
\left\{a^{i}, b^{i}\right\} & \Rightarrow_{N-1,2^{N-1}} & \{c, d\}, \quad i=1, \ldots, N-1, \\
\{a, b\} & \Rightarrow_{N-1,2^{N-1}} & \{c, d\} .
\end{array}
$$

Choose distinct $c=c_{0}, c_{1}, \ldots, c_{m}=d$ so that $\{a, b\} \rightarrow_{N-1}\left\{c_{j}, c_{j+1}\right\}$ for all $j<m$. Choose $(N-1)$-translations $f_{0}, \ldots, f_{m-1}$ so that $\left\{c_{j}, c_{j+1}\right\}=\left\{f_{j}(a), f_{j}(b)\right\}$ for all $j<m$. For each $j<m$ define a sequence $T_{j}$ from $c_{j}$ to $c_{j+1}$ by applying $f_{j}$ to $S_{N}$ or its reverse; that is,

$$
T_{j}= \begin{cases}\left(f_{j}\left(a_{0}^{N}\right), f_{j}\left(a_{1}^{N}\right), \ldots, f_{j}\left(a_{\lambda(N)}^{N}\right)\right) & \text { if }\left(f_{j}(a), f_{j}(b)\right)=\left(c_{j}, c_{j+1}\right), \\ \left(f_{j}\left(a_{\lambda(N)}^{N}\right), \ldots, f_{j}\left(a_{1}^{N}\right), f_{j}\left(a_{0}^{N}\right)\right) & \text { if }\left(f_{j}(a), f_{j}(b)\right)=\left(c_{j+1}, c_{j}\right) .\end{cases}
$$

Let $T$ be the sequence from $c$ to $d$ formed by concatenating $T_{0}, \ldots, T_{m-1}$. By Lemma 3.2 there must exist $\{u, v\} \in A^{(2)}$ and a link $\{r, s\}$ in $T$ such that $r \neq s$, $\{r, s\} \Rightarrow_{1,2}\{u, v\}$ and $\{c, d\} \Rightarrow_{1,2}\{u, v\}$. By construction, the link $\{r, s\}$ must be of the form $\left\{f_{j}\left(a_{k}^{N}\right), f_{j}\left(a_{k+1}^{N}\right)\right\}$ for some $j<m$ and $k<\lambda(N)$. Rename $\left\{a_{k}^{N}, a_{k+1}^{N}\right\}$ as $\left\{a^{N}, b^{N}\right\}$, our chosen key link of $S_{N}$. Then

$$
\begin{aligned}
& \left\{a^{i}, b^{i}\right\} \quad \Rightarrow_{N-1,2^{N-1}} \quad\{c, d\} \quad \Rightarrow_{1,2} \quad\{u, v\}, \quad i=1, \ldots, N-1, \\
& \{a, b\} \Rightarrow_{N-1,2^{N-1}} \quad\{c, d\} \Rightarrow_{1,2} \quad\{u, v\}, \\
& \left\{a^{N}, b^{N}\right\} \quad \rightarrow_{N-1} \quad\{r, s\} \quad \Rightarrow_{1,2} \quad\{u, v\} .
\end{aligned}
$$

Thus $\left\{a^{i}, b^{i}\right\}(1 \leq i \leq N)$ and $\{u, v\}$ have the desired properties. 
Corollary 3.4. With the same hypotheses as in Lemma 3.2, suppose $\mathbf{A} \in \mathcal{V}$, $\left\{a_{1}, b_{1}\right\}, \ldots,\left\{a_{N}, b_{N}\right\},\{u, v\} \in A^{(2)}$ and $n>0$ are such that $\left\{a_{i}, b_{i}\right\} \Rightarrow_{n}\{u, v\}$ for all $i=1, \ldots, N$. Then there exist $\left\{r_{i}, s_{i}\right\} \in A^{(2)}(1 \leq i \leq N)$ and $\left\{u^{\prime}, v^{\prime}\right\} \in A^{(2)}$ such that $\left\{a_{i}, b_{i}\right\} \rightarrow_{n}\left\{r_{i}, s_{i}\right\} \Rightarrow_{N, 2^{N}}\left\{u^{\prime}, v^{\prime}\right\}$ for all $i=1, \ldots, N$, and $\{u, v\} \Rightarrow_{N, 2^{N}}$ $\left\{u^{\prime}, v^{\prime}\right\}$. In particular, $\left\{a_{i}, b_{i}\right\} \Rightarrow_{n+N, 2^{N}}\left\{u^{\prime}, v^{\prime}\right\}$ for all $i$.

Proof. For each $i=1, \ldots, N$ choose a sequence $S_{i}=\left(u_{0}^{i}, u_{1}^{i}, \ldots, u_{\lambda(i)}^{i}\right)$ from $u$ to $v$ so that $\left\{a_{i}, b_{i}\right\} \rightarrow_{n}\left\{u_{j}^{i}, u_{j+1}^{i}\right\}$ for all $i=1, \ldots, N$ and all $j<\lambda(i)$. Apply the previous lemma to the sequences $S_{1}, \ldots, S_{N}$.

\section{THE MAIN THEOREM}

Theorem 4.1. Suppose $\mathcal{V}$ is a congruence meet-semidistributive variety in a finite language. If $\mathcal{V}$ contains arbitrarily large finite SI's, then $\mathcal{V}$ contains an infinite SI.

The main step in the proof is to show the following:

Lemma 4.2. Suppose $\mathcal{V}$ is a variety satisfying the hypotheses of Lemma 3.2. There is a function $g:(\omega \backslash\{0,1\}) \rightarrow \omega$ such that the following is true: if $\mathbf{A} \in \mathcal{V}$ is SI with monolith $\mu$, if $(c, d) \in \mu \backslash 0_{A}$, and if $2 \leq m<\omega$ with $|A| \geq m$, then there exist $S \subseteq A$ and $\{u, v\} \in A^{(2)}$ such that $|S|=m$ and $S^{(2)} \Rightarrow_{g(m)}\{u, v\}$ and $\{c, d\} \Rightarrow_{g(m)}\{u, v\}$.

Note that if $N(m)=m(m-1) / 2+1, f(m)=g(m)+N(m)$ and $h(m)=2^{N(m)}$, then a consequence of Lemma 4.2 and Corollary 3.4 is:

Corollary 4.3. Suppose $\mathcal{V}$ is a variety satisfying the hypotheses of Lemma 3.2. If $\mathbf{A} \in \mathcal{V}$ is SI with monolith $\mu$, if $(c, d) \in \mu \backslash 0_{A}$, and if $2 \leq m<\omega$ with $|A| \geq m$, then there exist $S \subseteq A$ and $\{u, v\} \in A^{(2)}$ such that $|S|=m$ and $S^{(2)} \Rightarrow_{f(m), h(m)}\{u, v\}$ and $\{c, d\} \Rightarrow_{f(m), h(m)}\{u, v\}$.

Proof of Theorem 4.1. $\mathcal{V}$ is term-equivalent to a variety in a finite language which satisfies the hypotheses of Lemma 3.2, so we may as well assume that $\mathcal{V}$ already satisfies these hypotheses. Fix $m \geq 2$ and choose an SI $\mathbf{A} \in \mathcal{V}$ satisfying $|A| \geq m$. Let $\mu$ be the monolith of $\mathbf{A}$ and choose $\left(c_{m}, d_{m}\right) \in \mu \backslash 0_{A}$. By Corollary 4.3 there exist a set $S_{m} \subseteq A$ and a pair $\{u, v\} \in A^{(2)}$ such that $\left|S_{m}\right|=m$ and $S_{m}^{(2)} \Rightarrow_{f(m), h(m)}$ $\{u, v\}$ and $\left\{c_{m}, d_{m}\right\} \Rightarrow_{f(m), h(m)}\{u, v\}$. Since $(u, v) \in \mathrm{Cg}^{\mathbf{A}}\left(c_{m}, d_{m}\right)=\mu$ and $u \neq v$ we have $(u, v) \in \mu \backslash 0_{A}$. Define $\left(c_{m-1}, d_{m-1}\right)=(u, v)$, and repeat the above argument with $m-1$ in place of $m$. Continuing in this way we will eventually get sets $S_{2}, \ldots, S_{m} \subseteq A$ and pairs $\left(c_{1}, d_{1}\right), \ldots,\left(c_{m}, d_{m}\right) \in \mu \backslash 0_{A}$ satisfying

(1) $\left\{c_{m}, d_{m}\right\} \Rightarrow_{f(m), h(m)}\left\{c_{m-1}, d_{m-1}\right\} \Rightarrow_{f(m-1), h(m-1)} \cdots\left\{c_{2}, d_{2}\right\} \Rightarrow_{f(2), h(2)}$ $\left\{c_{1}, d_{1}\right\}$,

(2) $\left|S_{k}\right|=k$ and $S_{k}^{(2)} \Rightarrow_{f(k), h(k)}\left\{c_{k-1}, d_{k-1}\right\}$ for $k=2, \ldots, m$.

It follows that $S_{k}^{(2)} \Rightarrow_{F(k), H(k)}\left\{c_{1}, d_{1}\right\}$ where $F(k)=\sum_{i=2}^{k} f(i)$ and $H(k)=$ $\prod_{i=2}^{k} h(i)$.

Since $\mathcal{V}$ is of finite type, for each $k \geq 2$ there is a first-order formula $\pi_{k}(x, y, z, w)$ defining the relation $\{x, y\} \Rightarrow_{F(k), H(k)}\{z, w\}$ in $\mathcal{V}$. Let $\mathrm{c}, \mathrm{d}, \mathrm{a}_{i}^{k}$ be new constant symbols (for $k \geq 2$ and $1 \leq i \leq k$ ). Let $T$ be the first-order theory consisting of the theory of $\mathcal{V}$ plus the sentences $\mathrm{c} \neq \mathrm{d}$ and $\pi_{k}\left(\mathrm{a}_{i}^{k}, \mathrm{a}_{j}^{k}, \mathrm{c}, \mathrm{d}\right)$ for all $1 \leq i<j \leq k$. A model of $T$ consists of a member $\mathbf{B}$ of $\mathcal{V}$ plus named subsets $S_{k}=\left\{a_{1}^{k}, \ldots, a_{k}^{k}\right\}(k \geq$ 2 ) and distinct elements $c, d$ such that $\left\{a_{i}^{k}, a_{j}^{k}\right\} \Rightarrow_{F(k), H(k)}\{c, d\}$ for $1 \leq i<j \leq k$. $T$ is finitely satisfiable by the previous discussion, so has a model $\left\langle\mathbf{B}, c, d,\left(a_{i}^{k}\right)\right\rangle$ by 
the compactness theorem of first-order logic. Let $\theta$ be a congruence of $\mathbf{B}$ which is maximal with respect to satisfying $(c, d) \notin \theta$. Then $\mathbf{B} / \theta$ is an SI in $\mathcal{V}$. Moreover, $(c / \theta, d / \theta) \in \operatorname{Cg}^{\mathbf{B} / \theta}\left(a_{i}^{k} / \theta, a_{j}^{k} / \theta\right)$, and hence $a_{i}^{k} / \theta \neq a_{j}^{k} / \theta$ for all $1 \leq i<j \leq k$, since the formulas $\pi_{k}\left(\mathrm{a}_{i}^{k}, \mathrm{a}_{j}^{k}, \mathrm{c}, \mathrm{d}\right)$ are preserved in passing to the quotient algebra. This proves that $|B / \theta| \geq\left|S_{k} / \theta\right|=k$ for every $k$, and hence $\mathbf{B} / \theta$ is infinite.

It remains to prove Lemma 4.2. This will be done in the next section.

\section{Proof of Lemma 4.2}

Throughout this section we assume that $\mathcal{V}$ is a variety satisfying the hypotheses of Lemma 3.2, that $2 \leq m<\omega$, that $\mathbf{A} \in \mathcal{V}$ is SI with monolith $\mu$ and with $|A| \geq m$, and that $(c, d) \in \mu \backslash 0_{A}$. We have claimed that there is a positive integer $g(m)$, depending on $m$ but not on $\mathbf{A}$ or $(c, d)$, for which we are guaranteed sets $S \subseteq A$ and $\{u, v\} \in A^{(2)}$ such that $|S|=m$ and $S \Rightarrow_{g(m)}\{u, v\}$ and $\{c, d\} \Rightarrow_{g(m)}\{u, v\}$. In fact, we provide estimates showing that $g(m)=2^{6 m-6}$ is sufficiently large.

For $n, k \geq 0$ let $C(n, k)$ denote the binomial coefficient, and define the following parameters:

$$
\begin{aligned}
M & =C(2 m-2, m-1)-1, \\
N & =C(M+1,2), \\
d & =3 N+4, \\
\ell & =d M, \\
g & =\ell\left(\left\lceil\log _{2} C(m, 2)\right\rceil+1\right), \\
p & =\ell-2 N-2, \\
q & =N+1 .
\end{aligned}
$$

As in $[1$, p. 228], the following inequalities

$$
\begin{aligned}
C(2 m-2, m-1) & \leq \frac{12}{11}\left(\frac{2^{2 m-2}}{(\pi(m-1))^{1 / 2}}\right), \\
d & \leq 2(M+1)^{2}, \\
1+\left\lceil\log _{2} C(m, 2)\right\rceil & \leq 2 \log _{2} m \leq 2(m-1), \\
\frac{4}{\pi^{3 / 2}}\left(\frac{12}{11}\right)^{3} & \leq 1,
\end{aligned}
$$

imply $g \leq 2^{6 m-6}$. We shall prove Lemma 4.2 with $g(m)=g$. It suffices to consider only $m \geq 3$.

Definition 5.1. Suppose $X \subseteq A^{(2)}$ and $k, n \geq 0$. We say that the members of $X$ are $(k, n)$-bounded over $\{c, d\}$ if there exists $\{u, v\} \in A^{(2)}$ such that $X \Rightarrow_{k}\{u, v\}$ and $\{c, d\} \Rightarrow_{n}\{u, v\}$.

Using this terminology, our goal is to prove the existence of $S \subseteq A$ with $|S|=m$ and such that the members of $S^{(2)}$ are $(g, g)$-bounded over $\{c, d\}$. Note also that if $\{r, s\} \in A^{(2)}$, then $\{r, s\}$ is $(0, n)$-bounded over $\{c, d\}$ if and only if $\{c, d\} \Rightarrow_{n}\{r, s\}$. Our proof of Lemma 4.2 breaks into three cases:

CASE 1. Any two $\{a, b\},\left\{a^{\prime}, b^{\prime}\right\} \in A^{(2)}$ are $(\ell, p)$-bounded over $\{c, d\}$.

CASE 2. There exists $\{r, s\} \in A^{(2)}$ which is $(0, \ell)$-bounded over $\{c, d\}$ but not $(q, p)$-bounded over $\{c, d\}$. 
CASE 3. For all $\{r, s\} \in A^{(2)}$, if $\{r, s\}$ is $(0, \ell)$-bounded over $\{c, d\}$, then $\{r, s\}$ is $(q, p)$-bounded over $\{c, d\}$; and there exist $\{a, b\},\left\{a^{\prime}, b^{\prime}\right\} \in A^{(2)}$ which are not $(\ell, p)$-bounded over $\{c, d\}$.

Proof of Lemma 4.2 in Case 1. We argue that for every set $S \subseteq A$ with $|S|=m$, the members of $S^{(2)}$ are $(g, g)$-bounded over $\{c, d\}$. To see this, choose an $S$ and partition $X_{0}=S^{(2)}$ into the minimum number possible of $\leq 2$-element subsets $T=\left\{\{a, b\},\left\{a^{\prime}, b^{\prime}\right\}\right\}$, and then for each such subset find some $\left\{u_{T}, v_{T}\right\}$ such that $T \Rightarrow_{\ell}\left\{u_{T}, v_{T}\right\}$. Let $X_{1}$ be the set of all such $\left\{u_{T}, v_{T}\right\}$. Note that $\left|X_{1}\right| \leq\left\lceil\frac{1}{2}\left|X_{0}\right|\right\rceil$. Repeat this process with $X_{1}$ to get $X_{2}, X_{3}, \ldots$ until at some $k \leq\left\lceil\log _{2}\left|X_{0}\right|\right\rceil$ we have $\left|X_{k}\right|=1$. Now find $\{u, v\} \in A^{(2)}$ such that $X_{k} \Rightarrow_{\ell}\{u, v\}$ and $\{c, d\} \Rightarrow_{p}$ $\{u, v\}$. Then working our way back through the construction, and noting that $g \geq \ell(k+1) \geq p$, we see that $S^{(2)} \Rightarrow_{g}\{u, v\}$ and $\{c, d\} \Rightarrow_{g}\{u, v\}$.

Proof of Lemma 4.2 in Case 2. Choose $\{r, s\} \in A^{(2)}$ which is $(0, \ell)$-bounded over $\{c, d\}$ but not $(q, p)$-bounded over $\{c, d\}$. Thus $\{c, d\} \Rightarrow_{\ell}\{r, s\}$. By Corollary 3.4 there exist $\left\{r_{1}, s_{1}\right\},\{u, v\} \in A^{(2)}$ such that $\{c, d\} \rightarrow_{\ell}\left\{r_{1}, s_{1}\right\} \Rightarrow_{1}\{u, v\}$ and $\{r, s\} \Rightarrow_{1}\{u, v\}$. Since $l=d M$, we can choose $\left\{a_{i}, b_{i}\right\} \in A^{(2)}(0 \leq i \leq M)$ such that $\{c, d\}=\left\{a_{0}, b_{0}\right\} \rightarrow_{d}\left\{a_{1}, b_{1}\right\} \rightarrow_{d} \cdots \rightarrow_{d}\left\{a_{M-1}, b_{M-1}\right\} \rightarrow_{d}\left\{a_{M}, b_{M}\right\}=\left\{r_{1}, s_{1}\right\}$. What is important is that for each $j$,

$$
\{c, d\} \rightarrow_{d j}\left\{a_{j}, b_{j}\right\} \rightarrow_{d(M-j)}\left\{r_{1}, s_{1}\right\} .
$$

For each $j \neq 0$ choose a $d(M-j)$-translation $f_{j}$ which witnesses $\left\{a_{j}, b_{j}\right\} \rightarrow_{d(M-j)}$ $\left\{r_{1}, s_{1}\right\}$. We assume that the elements have been named so that $f_{j}\left(a_{j}\right)=r_{1}$ and $f_{j}\left(b_{j}\right)=s_{1}$.

Recall that $\left\{r_{1}, s_{1}\right\} \Rightarrow_{1}\{u, v\}$. Choose $t<\omega$, elements $u_{0}, \ldots, u_{t} \in A$ and basic translations $g_{0}, \ldots, g_{t-1}$ such that $u_{0}=u, u_{t}=v$, and $\left\{g_{k}\left(r_{1}\right), g_{k}\left(s_{1}\right)\right\}=$ $\left\{u_{k}, u_{k+1}\right\}$ for $k<t$.

For $0 \leq i<j \leq M$ and $0 \leq k<t$ let $R_{i j}$ be the sequence from $r_{1}$ to $s_{1}$ obtained by applying $f_{j}$ to $\left(a_{j}, a_{i}, b_{i}, b_{j}\right)$, and let $S_{i j k}$ be the sequence from $u_{k}$ to $u_{k+1}$ obtained by applying $g_{k}$ to $R_{i j}$ or its reverse. Let $S_{i j}$ be the sequence obtained by concatenating the sequences $S_{i j 0}, \ldots, S_{i j t-1}$. Thus $S_{i j}$ is a sequence from $u$ to $v$ such that for each adjacent pair $\{x, y\}$ with $x \neq y$, one of the following holds:

(a) $\left\{a_{i}, a_{j}\right\} \rightarrow d(M-j)+1\{x, y\}$,

(m) $\left\{a_{i}, b_{i}\right\} \rightarrow_{d(M-j)+1}\{x, y\}$, "the middle case", or

(b) $\left\{b_{i}, b_{j}\right\} \rightarrow d(M-j)+1\{x, y\}$.

In all we get $C(M+1,2)=N$ such sequences from $u$ to $v$. By Lemma 3.3 there exist $\left\{u_{1}, v_{1}\right\} \in A^{(2)}$ and 'key links' $\left\{x_{i j}, y_{i j}\right\}$ for $S_{i j}, 0 \leq i<j \leq M$, such that $\left\{x_{i j}, y_{i j}\right\} \Rightarrow_{N}\left\{u_{1}, v_{1}\right\}$, and $\{u, v\} \Rightarrow_{N}\left\{u_{1}, v_{1}\right\}$. Let $\left\{u_{1}, v_{1}\right\}$ and the key links be chosen and fixed.

We claim that for no $i$ and $j$ with $0 \leq i<j \leq M$ can the key link $\{x, y\}=$ $\left\{x_{i j}, y_{i j}\right\}$ in $S_{i j}$ be as described in "the middle case". The reason for this is that if $\left\{a_{i}, b_{i}\right\} \rightarrow_{d(M-j)+1}\left\{x_{i j}, y_{i j}\right\}$, then $\{c, d\} \rightarrow_{\ell-d+1}\left\{x_{i j}, y_{i j}\right\}\left(\right.$ as $\{c, d\} \rightarrow_{d i}\left\{a_{i}, b_{i}\right\}$ and $d i+d(M-j) \leq d(M-1)=\ell-d)$, and so

$$
\text { and } \begin{aligned}
& \{c, d\} \rightarrow_{\ell-d+1}\left\{x_{i j}, y_{i j}\right\} \Rightarrow_{N}\left\{u_{1}, v_{1}\right\} \\
& \{r, s\} \Rightarrow_{1}\{u, v\} \Rightarrow_{N}\left\{u_{1}, v_{1}\right\} .
\end{aligned}
$$


Thus $\{c, d\} \Rightarrow_{p}\left\{u_{1}, v_{1}\right\}$ and $\{r, s\} \Rightarrow_{q}\left\{u_{1}, v_{1}\right\}$, since $\ell-d+1+N \leq p$ and $1+N=q$. But then $\{r, s\}$ would be $(q, p)$-bounded over $\{c, d\}$, contradicting the assumption of Case 2 .

Color the complete graph on the vertices $\{0,1, \ldots, M\}$ according to the rule: edge $\{i, j\}$ gets color a if the key link in $S_{i j}$ is as in item (a); otherwise this edge gets color b. Since $M+1=C(2 m-2, m-1)$ is at least as large as the Ramsey number for a complete monochromatic subgraph of $m$ vertices in 2-colored complete graphs, there is an $m$-element subset $J \subseteq\{0,1, \ldots, M\}$ such that we are in item (a) for all $i, j \in J$ with $i<j$, or in item (b) for all $i, j \in J$ with $i<j$. In the former case take $S=\left\{a_{j}: j \in J\right\}$; in the latter take $S=\left\{b_{j}: j \in J\right\}$. Assuming that $S=\left\{a_{j}: j \in J\right\}$, then for all $i<j$ in $J$ we have

$$
\begin{array}{ll} 
& \left\{a_{i}, a_{j}\right\} \rightarrow_{d(M-j)+1}\left\{x_{i j}, y_{i j}\right\} \Rightarrow_{N}\left\{u_{1}, v_{1}\right\} \\
\text { and } & \{c, d\} \Rightarrow_{\ell}\{r, s\} \Rightarrow_{1}\{u, v\} \Rightarrow_{N}\left\{u_{1}, v_{1}\right\} .
\end{array}
$$

Since this holds for all $i<j$ in $J$, and since $d(M-j)+1+N \leq \ell+1+N \leq g$ (as $m \geq 3)$, we get $S^{(2)} \Rightarrow_{g}\left\{u_{1}, v_{1}\right\}$ and $\{c, d\} \Rightarrow_{g}\left\{u_{1}, v_{1}\right\}$.

Proof of Lemma 4.2 in Case 3. Choose $\{a, b\},\left\{a^{\prime}, b^{\prime}\right\} \in A^{(2)}$ which are not $(\ell, p)$ bounded over $\{c, d\}$. Because $\mathbf{A}$ is subdirectly irreducible and $(c, d) \in \mu \backslash 0_{A}$, there exists $n<\omega$ such that $\{a, b\} \Rightarrow_{n}\{c, d\}$ and $\left\{a^{\prime}, b^{\prime}\right\} \Rightarrow_{n}\{c, d\}$. That is, $\{a, b\}$ and $\left\{a^{\prime}, b^{\prime}\right\}$ are $(n, 0)$-bounded over $\{c, d\}$ for some $n$, and therefore are $(n, p)$-bounded over $\{c, d\}$ for some $n$. Pick $n$ minimum such that $\{a, b\}$ and $\left\{a^{\prime}, b^{\prime}\right\}$ are $(n, p)$ bounded over $\{c, d\}$; by assumption, $n>\ell$. Also pick $\left\{u_{0}, v_{0}\right\} \in A^{(2)}$ such that $\{a, b\} \Rightarrow_{n}\left\{u_{0}, v_{0}\right\},\left\{a^{\prime}, b^{\prime}\right\} \Rightarrow_{n}\left\{u_{0}, v_{0}\right\}$, and $\{c, d\} \Rightarrow_{p}\left\{u_{0}, v_{0}\right\}$.

Corollary 3.4 applied to the two instances of $\Rightarrow_{n}$ yields $\left\{r_{1}, s_{1}\right\},\left\{r_{1}^{\prime}, s_{1}^{\prime}\right\},\{u, v\} \in$ $A^{(2)}$ such that

$$
\begin{aligned}
& \{a, b\} \rightarrow_{n}\left\{r_{1}, s_{1}\right\} \Rightarrow_{2}\{u, v\} \\
& \left\{a^{\prime}, b^{\prime}\right\} \rightarrow_{n}\left\{r_{1}^{\prime}, s_{1}^{\prime}\right\} \Rightarrow_{2}\{u, v\} \\
& \left\{u_{0}, v_{0}\right\} \Rightarrow_{2}\{u, v\} .
\end{aligned}
$$

Since $n>\ell$, we can choose $\left\{a_{0}, b_{0}\right\},\left\{a_{0}^{\prime}, b_{0}^{\prime}\right\} \in A^{(2)}$ such that

$$
\begin{array}{rllll}
\{a, b\} & \rightarrow_{n-\ell} & \left\{a_{0}, b_{0}\right\} & \rightarrow_{\ell} & \left\{r_{1}, s_{1}\right\}, \\
\left\{a^{\prime}, b^{\prime}\right\} & \rightarrow_{n-\ell} & \left\{a_{0}^{\prime}, b_{0}^{\prime}\right\} & \rightarrow_{\ell} & \left\{r_{1}^{\prime}, s_{1}^{\prime}\right\} .
\end{array}
$$

Choose $\left\{a_{i}, b_{i}\right\},\left\{a_{i}^{\prime}, b_{i}^{\prime}\right\} \in A^{(2)}(1 \leq i \leq M)$ such that

$$
\left\{a_{0}, b_{0}\right\} \rightarrow_{d}\left\{a_{1}, b_{1}\right\} \rightarrow_{d} \cdots \rightarrow_{d}\left\{a_{M-1}, b_{M-1}\right\} \rightarrow_{d}\left\{a_{M}, b_{M}\right\}=\left\{r_{1}, s_{1}\right\}
$$

and similarly for the primed pairs. As in Case 2, for $0 \leq i<j \leq M$ we can produce a sequence $S_{i j}$ from $u$ to $v$ with the property that for each adjacent pair $\{x, y\}$ in the sequence with $x \neq y$, either

(a) $\left\{a_{i}, a_{j}\right\} \rightarrow d(M-j)+2\{x, y\}$

(m) $\left\{a_{i}, b_{i}\right\} \rightarrow d(M-j)+2\{x, y\}$, or

(b) $\left\{b_{i}, b_{j}\right\} \rightarrow d(M-j)+2\{x, y\}$.

Similarly, for $0 \leq i<j \leq M$ we can obtain a sequence $S_{i j}^{\prime}$ from $u$ to $v$ such that for each adjacent pair $\left\{x^{\prime}, y^{\prime}\right\}$ with $x^{\prime} \neq y^{\prime}$, one of the following holds:

$\left(\mathrm{a}^{\prime}\right)\left\{a_{i}^{\prime}, a_{j}^{\prime}\right\} \rightarrow d(M-j)+2\left\{x^{\prime}, y^{\prime}\right\}$,

$\left(\mathrm{m}^{\prime}\right)\left\{a_{i}^{\prime}, b_{i}^{\prime}\right\} \rightarrow_{d(M-j)+2}\left\{x^{\prime}, y^{\prime}\right\}$, or

$\left(\mathrm{b}^{\prime}\right)\left\{b_{i}^{\prime}, b_{j}^{\prime}\right\} \rightarrow_{d(M-j)+2}\left\{x^{\prime}, y^{\prime}\right\}$. 
In all we get $2 \cdot C(M+1,2)=2 N$ sequences from $u$ to $v$. By Lemma 3.3 there exist $\left\{u_{1}, v_{1}\right\} \in A^{(2)}$ and 'key links' $\left\{x_{i j}, y_{i j}\right\}$ for $S_{i j}$ and $\left\{x_{i j}^{\prime}, y_{i j}^{\prime}\right\}$ for $S_{i j}^{\prime}, 0 \leq i<j \leq$ $M$, such that $\left\{x_{i j}, y_{i j}\right\} \Rightarrow_{2 N}\left\{u_{1}, v_{1}\right\}$ and similarly for the primed key links, and $\{u, v\} \Rightarrow_{2 N}\left\{u_{1}, v_{1}\right\}$. Let $\left\{u_{1}, v_{1}\right\}$ and the key links be chosen and fixed. Note that

$$
\{c, d\} \Rightarrow_{p}\left\{u_{0}, v_{0}\right\} \Rightarrow_{2}\{u, v\} \Rightarrow_{2 N}\left\{u_{1}, v_{1}\right\} .
$$

Since $p+2+2 N=\ell$, we get $\{c, d\} \Rightarrow \ell\left\{u_{1}, v_{1}\right\}$, i.e., $\left\{u_{1}, v_{1}\right\}$ is $(0, \ell)$-bounded over $\{c, d\}$. By the assumption of Case $3,\left\{u_{1}, v_{1}\right\}$ must be $(q, p)$-bounded over $\{c, d\}$; that is, there exists $\left\{u_{2}, v_{2}\right\} \in A^{(2)}$ such that $\left\{u_{1}, v_{1}\right\} \Rightarrow_{q}\left\{u_{2}, v_{2}\right\}$ and $\{c, d\} \Rightarrow_{p}\left\{u_{2}, v_{2}\right\}$.

Now suppose for some $S_{i j}$ that the key link $\{x, y\}=\left\{x_{i j}, y_{i j}\right\}$ is as described in item $(\mathrm{m})$. Then $\left\{a_{0}, b_{0}\right\} \rightarrow_{\ell-d+2}\left\{x_{i j}, y_{i j}\right\}$, and hence

$$
\{a, b\} \rightarrow_{n-\ell}\left\{a_{0}, b_{0}\right\} \rightarrow_{\ell-d+2}\left\{x_{i j}, y_{i j}\right\} \Rightarrow_{2 N}\left\{u_{1}, v_{1}\right\} \Rightarrow_{q}\left\{u_{2}, v_{2}\right\} .
$$

Since $n-d+2+2 N+q=n-1$, we get $\{a, b\} \Rightarrow_{n-1}\left\{u_{2}, v_{2}\right\}$. Likewise, if for some $S_{i^{\prime} j^{\prime}}^{\prime}$ the chosen key link is as described in item $\left(\mathrm{m}^{\prime}\right)$, then $\left\{a^{\prime}, b^{\prime}\right\} \Rightarrow_{n-1}\left\{u_{2}, v_{2}\right\}$. Recall that $\{c, d\} \Rightarrow_{p}\left\{u_{2}, v_{2}\right\}$ and that $\{a, b\},\left\{a^{\prime}, b^{\prime}\right\}$ are not $(n-1, p)$-bounded over $\{c, d\}$. Thus either item $(\mathrm{m})$ never occurs or item $\left(\mathrm{m}^{\prime}\right)$ never occurs. By symmetry we can assume that item $(\mathrm{m})$ never occurs, and henceforth ignore the primed elements. Thus for all $0 \leq i<j \leq M$, the chosen key link of $S_{i j}$ is as described in items (a) or (b). As in Case 2, Ramsey's theorem yields an $m$-element subset $J \subseteq\{0,1, \ldots, M\}$ such that either all sequences $S_{i j}$ with $i, j \in J$ and $i<j$ have their key links as in item (a), or all have their key links as in item (b). Again for concreteness let us assume that all have their key links as in item (a). Let $S=\left\{a_{j}: j \in J\right\}$. Then for all $i, j \in J$ with $i<j$,

$$
\left\{a_{i}, a_{j}\right\} \rightarrow_{d(M-j)+2}\left\{x_{i j}, y_{i j}\right\} \Rightarrow_{2 N}\left\{u_{1}, v_{1}\right\} .
$$

Since $d(M-j)+2+2 N \leq \ell+2+2 N \leq g$ (as $m \geq 3$ ), and since it was shown above that $\{c, d\} \Rightarrow_{\ell}\left\{u_{1}, v_{1}\right\}$, it follows that the members of $S^{(2)}$ are $(g, g)$-bounded over $\{c, d\}$, as required.

\section{REFERENCES}

[1] K. Baker, Finite equational bases for finite algebras in a congruence-distributive equational class, Adv. in Math. 24 (1977), 207-243. MR 56:5389

[2] G. Czédli, A characterization of congruence semi-distributivity, in Universal Algebra and Lattice Theory (Proc. Conf. Puebla, 1982), Springer Lecture Notes No. 1004, 1983. MR 85g:08006

[3] A. Foster and A. Pixley, Semi-categorical algebras. II, Math. Zeit. 85 (1964), 169-184. MR 29:5771

[4] D. Hobby and R. McKenzie, The Structure of Finite Algebras, Contemporary Mathematics 76, American Math. Soc. (Providence, RI), 1988. MR 89m:08001

[5] W. Hodges, Model Theory, Encyclopedia of Mathematics and its Applications 42, Cambridge University Press, 1993. MR 94e:03002

[6] K. Kaarli and A. Pixley, Affine complete varieties, Algebra Universalis 24 (1987), 74-90. MR 88k:08002

[7] K. Kearnes and Á. Szendrei, The relationship between two commutators, to appear in Internat. J. Algebra Comput.

[8] P. Lipparini, A characterization of varieties with a difference term, II: neutral = meet semidistributive, Canad. Math. Bull. 41 (1998), 318-327. CMP 98:16

[9] R. McKenzie, The residual bounds of finite algebras, Internat. J. Algebra Comput. 6 (1996), 1-28. MR 97e:08002a

[10] R. W. Quackenbush, Equational classes generated by finite algebras, Algebra Universalis 1 (1971), 265-266. MR 45:3295 
[11] W. Taylor, Residually small varieties, Algebra Universalis 2 (1972), 33-53. MR 47:3278

[12] R. Willard, A finite basis theorem for residually finite congruence meet-semidistributive varieties, to appear in J. Symbolic Logic.

Department of Mathematics, University of Louisville, Louisville, Kentucky 40292

E-mail address: kearnes@louisville.edu

Department of Pure Mathematics, University of Waterloo, Waterloo, Ontario, CANADA N2L 3G1

E-mail address: rdwillar@gillian.math.uwaterloo.ca 